\title{
Effects of Porphyromonas gingivalis lipopolysaccharide on the expression of key genes involved in cholesterol metabolism in macrophages
}

\author{
Fen $\mathrm{Liu}^{1}$, Yi Wang ${ }^{2}$, Jing $\mathrm{Xu}^{3}$, Fangqiang $\mathrm{Li}^{3}$, Rongdang $\mathrm{Hu}^{2}$, Hui Deng ${ }^{3}$
}

\author{
${ }^{1}$ Department of Histology and Embryology, Wenzhou Medical University, Wenzhou, \\ Zhejiang, China \\ ${ }^{2}$ Department of Orthodontics, School of Stomatology, Wenzhou Medical University, \\ Wenzhou, Zhejiang, China \\ ${ }^{3}$ Department of Periodontics, School of Stomatology, Wenzhou Medical University, \\ Wenzhou, Zhejiang, China
}

Submitted: 2 March 2014

Accepted: 27 June 2014

Arch Med Sci 2016; 12, 5: 959-967

DOI: 10.5114/aoms.2016.61909

Copyright $\odot 2016$ Termedia \& Banach

\section{Abstract}

Introduction: Cardiovascular diseases are positively correlated with periodontal disease. However, the molecular mechanisms linking atherosclerosis and periodontal infection are not clear. This study aimed to determine whether Porphyromonas gingivalis lipopolysaccharide (Pg-LPS) altered the expression of genes regulating cholesterol metabolism in macrophages in the presence of low-density lipoprotein (LDL).

Material and methods: THP-1-derived macrophages were exposed to different concentrations $(0.1,1,10 \mu \mathrm{g} / \mathrm{ml})$ of LPS in the presence of $50 \mu \mathrm{g} / \mathrm{ml}$ native LDL. Macrophages were also incubated with $1 \mu \mathrm{g} / \mathrm{ml}$ LPS for varying times $(0,24,48$, or $72 \mathrm{~h})$ in the presence of native LDL. Foam cell formation was determined by oil red $O$ staining and cholesterol content quantification. CD36, lectin-like oxidized LDL receptor-1 (LOX-1), ATP-binding cassette G1 (ABCG1), and acetyl CoA acyltransferase 1 (ACAT1) expression levels were measured by western blot and qRT-PCR.

Results: Foam cell formation was induced in a time- and concentration-dependent manner as assessed by both morphological and biochemical criteria. Pg-LPS caused downregulation of CD36 and ABCG1 but upregulation of ACAT1, while LOX-1 expression was not affected $(p=0.137)$.

Conclusions: Pg-LPS appears to be an important link in the development of atherosclerosis by mechanisms targeting cholesterol homeostasis, namely, excess cholesterol ester formation via ACAT1 and reduced cellular cholesterol efflux via ABCG1.

Key words: periodontitis, macrophages, CD36, acetyl CoA acyltransferase 1, ATP-binding cassette $\mathrm{G} 1$.

\section{Introduction}

In recent decades, infection and inflammation have been shown to be involved in atherosclerosis initiation and progression [1]. Periodontal disease is one of the most prevalent infections found in humans [2]. Data from a meta-analysis [3] suggested that periodontal disease is an independent risk factor for cardiovascular diseases. Porphyromonas gingivalis (Pg), a major pathogen in periodontitis, is found in athero-

\author{
Corresponding authors: \\ Hui Deng \\ Department of Periodontics \\ School of Stomatology \\ Wenzhou Medical University \\ No. 113 West Xueyuan Road \\ Wenzhou, Zhejiang, China \\ Phone/fax: \\ +86 577-88066062 \\ E-mail:dh0726@gmail.com \\ Rongdang $\mathrm{Hu}$ \\ Department of Orthodontics \\ School of Stomatology \\ Wenzhou Medical University \\ No. 113 West Xueyuan Road \\ Wenzhou, Zhejiang, China \\ Phone/fax: \\ +86 577-88855488 \\ E-mail: hurongdang@hotmail. \\ com
}


sclerotic plaques [4]. Moreover, Pg lipopolysaccharide (Pg-LPS) infection may exacerbate atherosclerosis pathogenesis [5]. These data suggest that Pg and Pg-LPS may invade the systemic bloodstream through the disrupted basement membrane, which is easily seen in severe periodontitis, thereby contributing to atherosclerosis progression.

The process of atherosclerosis begins with the recruitment of monocyte-derived macrophages into the arterial intima and their transformation into lipid-loaded foam cells through the uptake of modified low-density lipoprotein (LDL) [6]. An in vitro study [7] demonstrated that Pg and Pg-LPS can induce foam cell formation in murine macrophages, partly by promoting the association of LDL with macrophages and the modification of LDL, indicating a causal relationship between PgLPS and atherosclerosis.

Macrophage cholesterol homeostasis, which is regulated by cholesterol synthesis, influx, and efflux, plays a vital role in foam cell formation [8]. The influx pathway occurs by uptake of modified LDL mainly through scavenger receptors (SRs), among which SR-A and CD36 have been identified as being responsible for $75-90 \%$ of the uptake [9]. Additionally, lectin-like oxidized LDL receptor-1 (LOX-1), a new member of the scavenger receptor family, may also be involved in ox-LDL uptake and foam cell formation [10]. Upon excess free cholesterol (FC) delivery to the endoplasmic reticulum, FC is rapidly re-esterified by acetyl-CoA cholesterol acyltransferase 1 (ACAT1) and then stored in cytoplasmic lipid droplets, creating a characteristic "foamy" appearance [11]. Given that ACAT1-regulated re-esterification is initially beneficial for cells for preventing cell toxicity induced by FC, increased ACAT1 expression may lead to the transformation of macrophages into foam cells, accelerating atherosclerosis development in humans [12]. Apart from re-esterification, the FC efflux pathway is another major mechanism for removing cellular cholesterol. Cholesterol efflux to lipid-poor ApoA occurs specifically via ATP-binding cassette (ABC) transporter $\mathrm{G} 1$ (ABCG1) [13]. Pg-LPS alone has been demonstrated to induce foam cell formation by modifying cells at the level of gene expression $[14,15]$. However, Pg-LPS-induced foam cell formation results from increased LDL uptake [7]. Despite the extensive studies on these topics, the underlying mechanisms responsible for foam cell formation in cells infected with LPS in the presence of LDL are not yet clear.

Here, we investigated the effects of Pg-LPS infection on the expression levels of key proteins supporting foam cell formation in the presence of native LDL in LPS-treated macrophages.

\section{Material and methods}

\section{Cell culture}

Human THP-1 monocytes obtained from the cell bank of Shanghai Institutes for Biological Sciences were seeded in 6-well plates at a density of $1 \times 10^{5}$ cells per well and cultured in RPMI 1640 medium (Hyclone, USA) supplemented with $2 \mathrm{mM}$ L-glutamine, $10 \%$ fetal bovine serum (FBS), and antibiotics at $37^{\circ} \mathrm{C}$ and $5 \% \mathrm{CO}_{2}$. After the addition of $160 \mathrm{nmol} / \mathrm{l}$ phorbol 12-myristate 13-acetate (PMA) for 48 h, THP-1 cells were differentiated into macrophages for further studies.

\section{Pg-LPS infection of THP-1-derived macrophages}

Ultrapure LPS from Porphyromonas gingivalis (catalog \#tlrl-pglps, version \#10G20 MT) was purchased from InvivoGen (USA), and human LDL was purchased from Beijing Xiesheng Bio Company (China). To test the effects of Pg-LPS on foam cell formation, THP-1-derived macrophages were incubated with or without increasing concentrations of Pg-LPS $(0.1,1,10 \mu \mathrm{g} / \mathrm{ml})$ in the presence of LDL $(50 \mu \mathrm{g} / \mathrm{ml})$ for $48 \mathrm{~h}$. In our preliminary experiments, macrophages were cultured in the presence of $50 \mu \mathrm{g} / \mathrm{ml} \mathrm{LDL}$ alone, and no significant changes were found in the amount of Oil Red O-stained cells or in the content of cellular cholesterol after culture for as long as $72 \mathrm{~h}$ (data not shown). These data showed that LDL alone (without stimulation by LPS or PMA) did not induce foam cell formation. Therefore, cells were further stimulated with LDL $(50 \mu \mathrm{g} / \mathrm{ml})$ and Pg-LPS $(1 \mu \mathrm{g} / \mathrm{ml})$ at different time intervals $(0,24,48$, or $72 \mathrm{~h})$.

\section{Oil Red O staining}

THP-1-derived macrophages were washed twice with phosphate-buffered saline (PBS) and fixed in 4\% paraformaldehyde for $15 \mathrm{~min}$. Cells were then stained with Oil Red O (Sigma, USA) for 10 min and counterstained with hematoxylin for $1 \mathrm{~min}$. The intracellular lipid droplets were stained red, while cell nuclei were stained blue under light microscopy. Macrophages with 10 or more Oil Red O-positive lipid droplets were defined as foam cells according to morphological criteria [16]. The number of foam cells was scored in three fields per well, and the percentage of foam cells was calculated according to the total number of cells counted.

\section{Quantification of cellular cholesterol content}

To further measure the formation of foam cells according to biochemical criteria, the amounts of total cholesterol (TC) and FC were quantified using the zymochemistry method as previously de- 
scribed [17]. Briefly, cells from each sample were disrupted by ultrasonication. Then the TC and FC contents were determined with a standard curve and expressed as $\mu \mathrm{g}$ cholesterol/mg protein. The cholesterol ester (CE) content was calculated by subtracting FC from TC. Foam cells were defined when the ratio of CE to TC was over 50\% [18].

\section{MTT assay}

MTT assays were performed to determine the cytotoxicity of LPS in cells. Briefly, after $4 \mathrm{~h}$ of incubation with $20 \mu \mathrm{l}$ MTT ( $5 \mathrm{mg} / \mathrm{ml}$; Sigma, USA), culture medium was removed from each sample and dissolved in $150 \mu \mathrm{l}$ of DMSO (Sigma). The absorbance was measured at $490 \mathrm{~nm}$, and the viability of cells was calculated as the relative ratio of absorbance to that of control.

\section{RNA isolation and real-time RT-PCR}

Total RNA from each sample was isolated with TRIzol reagent (Invitrogen, USA) according to the manufacturer's instructions. cDNA was synthesized with M-MuLV transcriptase (Fermentas, Canada) in a $20-\mu \mathrm{l}$ mixture containing $2 \mu \mathrm{g}$ of RNA. The sequences of forward and reverse primers are given in Table I. Real-time PCR was then performed using SYBR premix EX Taq II buffer (Takara, Japan) on a LightCycler 480 system (Roche, USA). The amplification was carried out as follows: one cycle of $95^{\circ} \mathrm{C}$ for $30 \mathrm{~s}$, followed by 40 cycles of $5 \mathrm{~s}$ at $95^{\circ} \mathrm{C}$ for denaturing, $20 \mathrm{~s}$ at $60^{\circ} \mathrm{C}$ for annealing, and $20 \mathrm{~S}$ at $75^{\circ} \mathrm{C}$ for extension. The relative levels of CD36, LOX-1, ACAT1, and ABCG1 were calculated according to the formula $2^{-\Delta \Delta C t}$. The data are presented as fold changes in target gene expression normalized to $\beta$-actin and relative to the untreated control.

\section{Western blot analysis}

After the cells were washed twice with PBS, they were lysed in lysis buffer (Beyotime, China) and incubated on ice for $30 \mathrm{~min}$. The lysates were centrifuged at $12,000 \mathrm{rpm}$ for $10 \mathrm{~min}$ at $4^{\circ} \mathrm{C}$. The protein concentration was determined with the BCA assay (Beyotime). Twenty micrograms of protein from each sample was separated by electrophoresis on $10 \%$ sodium dodecyl sulfate (SDS) polyacrylamide gels and transferred to polyvinylidene difluoride (PVDF) membranes (Bio-Rad, Hercules, CA, USA). The membranes were then probed with rabbit polyclonal anti-CD36 (1 : 2000), anti-LOX-1 (1 : 2000), anti-ACAT1 (1 : 1500), anti-ABCG 1 (1 : 2000), or anti- $\beta$-actin (1 : 2000) antibodies (Proteintech, China) overnight at $4^{\circ} \mathrm{C}$. Subsequently, membranes were incubated with horseradish peroxidase (HRP)-conjugated secondary antibodies (1 : 2000; Proteintech Inc) for $60 \mathrm{~min}$ at room
Table I. Primer sequences of target genes and $\beta$-actin for real-time PCR

\begin{tabular}{|c|c|}
\hline Target gene & Sequences \\
\hline \multirow[t]{2}{*}{$\beta$-actin } & F 5'-CATTGCCGACAGGATGCAG-3' \\
\hline & R 5'-CTCGTCATACTCCTGCTTGCTG-3' \\
\hline \multirow[t]{2}{*}{ CD36 } & F 5'-GGTGATGATGGAGAATAAGCC-3' \\
\hline & R 5'-AAGAGCCCAGAGTCGGAGTTG-3' \\
\hline \multirow[t]{2}{*}{ LOX-1 } & F 5'-CTTGCTCGGAAGCTGAATG-3' \\
\hline & R 5'-CCGTCCTCCCAGAGCCAT-3' \\
\hline \multirow[t]{2}{*}{ ACAT1 } & F 5'-TTCTCATCCGCTGATCCGTT-3' \\
\hline & R 5'-TAGCGACATAACCCCATCTTACAG-3' \\
\hline \multirow[t]{2}{*}{ ABCG 1} & F 5'-CATTGCCGACAGGATGCAG-3' \\
\hline & R 5'-CTCGTCATACTCCTGCTTGCTG-3' \\
\hline
\end{tabular}

temperature. Immunoreactivity was detected by enhanced chemiluminescence $(E C L)$. The relative expression levels of target genes were normalized to the expression of $\beta$-actin.

\section{Statistical analysis}

All experiments were repeated at least three times, and data were presented as means \pm standard deviations (SDs). Student's $t$ tests were used for direct comparisons between two groups, while one-way analysis of variance (ANOVA) was used for comparisons of three or more groups, followed by Tukey's post hoc test. Differences with $p$-values of less than 0.05 (two-tailed) were considered statistically significant.

\section{Results}

Pg-LPS induced foam cell formation in a concentration- and time-dependent manner, according to morphological criteria

After treatment with $0.1 \mu \mathrm{g} / \mathrm{ml} \mathrm{Pg-LPS}$ and LDL (Figure $1 \mathrm{~B}$ ), foam cell formation was minimal, but was obviously higher than that obtained for the control (LDL alone), as demonstrated by Oil Red O staining of lipid droplets (Figure 1 A). However, in cells treated with higher Pg-LPS and LDL concentrations, large lipid droplet masses were observed (Figures $1 C$ and D). The number of foam cells significantly increased in all the Pg-LPS treated groups (Figure $1 \mathrm{I}$ ). These results indicated that LPS exposure caused foam cell formation in a concentration-dependent manner.

Next, macrophages were stimulated with $1 \mu \mathrm{g} /$ $\mathrm{ml} \mathrm{Pg-LPS}$ in the presence of LDL for increasing times. Compared to the results for uninfected macrophages (Figure $1 \mathrm{E}$ ), increasing numbers of Oil Red O-stained cells were observed with longer durations of treatment (Figures $1 \mathrm{~F}-\mathrm{H}$ ), sug- 
gesting that Pg-LPS induced foam cell formation in a time-dependent manner. Furthermore, the number of foam cells increased significantly with increase in infection times (Figure $1 \mathrm{~J}$ ). MTT assays showed that the cell viability was greater than $95 \%$ at the highest dose and longest treatment time (data not shown), demonstrating that the treatment did not alter macrophage viability.

\section{Pg-LPS induced foam cell formation}

in a concentration- and time-dependent manner, according to biochemical criteria

Given that excessive CE accounted for the elevated amount of lipid droplets, the cellular CE content was measured to investigate foam cell formation according to biochemical criteria. Incubation of LDL-treated macrophages with Pg-LPS caused a concentration-dependent increase in $\mathrm{CE}$ and TC accumulation (Figure $2 \mathrm{~A}$ ). Moreover, treatment with 1 or $10 \mu \mathrm{g} / \mathrm{ml}$ LPS significantly induced foam cell formation (Figure 2 B), as assessed when using CE/TC > 50\% for identification of foam cells [18]. As shown in Figures 2 C, Pg-LPS exposure induced a time-dependent increase in CE and $\mathrm{TC}$ content, and the CE/TC ratio was greater than $50 \%$ after $24 \mathrm{~h}$ of LPS exposure (Figure $2 \mathrm{D}$ ), which indicated foam cell formation according to biochemical criteria.

\section{CD36 was downregulated by Pg-LPS treatment}

Since CD36 and LOX-1 regulate lipoprotein uptake, CD36 and LOX-1 mRNA and protein expression levels were measured to investigate the cholesterol influx pathway during Pg-LPS exposure in the presence of LDL. Pg-LPS treatment downregulated CD36 mRNA expression in a concentration-dependent manner, nearly reaching less than $50 \%$ of that of the control $(10 \mu \mathrm{g} / \mathrm{ml}$; Figure $3 \mathrm{~A})$. Western blot analysis (Figure $3 \mathrm{~B}$ ) revealed similar downregulation of CD36, with a dramatic $90 \%$ drop in comparison with that of the control at the highest concentration $(10 \mu \mathrm{g} / \mathrm{ml})$. No significant changes were found in the mRNA or protein expression of LOX-1, another SR family member, even when cells were exposed to the highest concentration of Pg-LPS applied in our study $(p>0.05)$.

Because of treatment with Pg-LPS $(1 \mu \mathrm{g} / \mathrm{ml})$ in the presence of LDL, we observed a significant time-dependent decrease in CD36 expression.
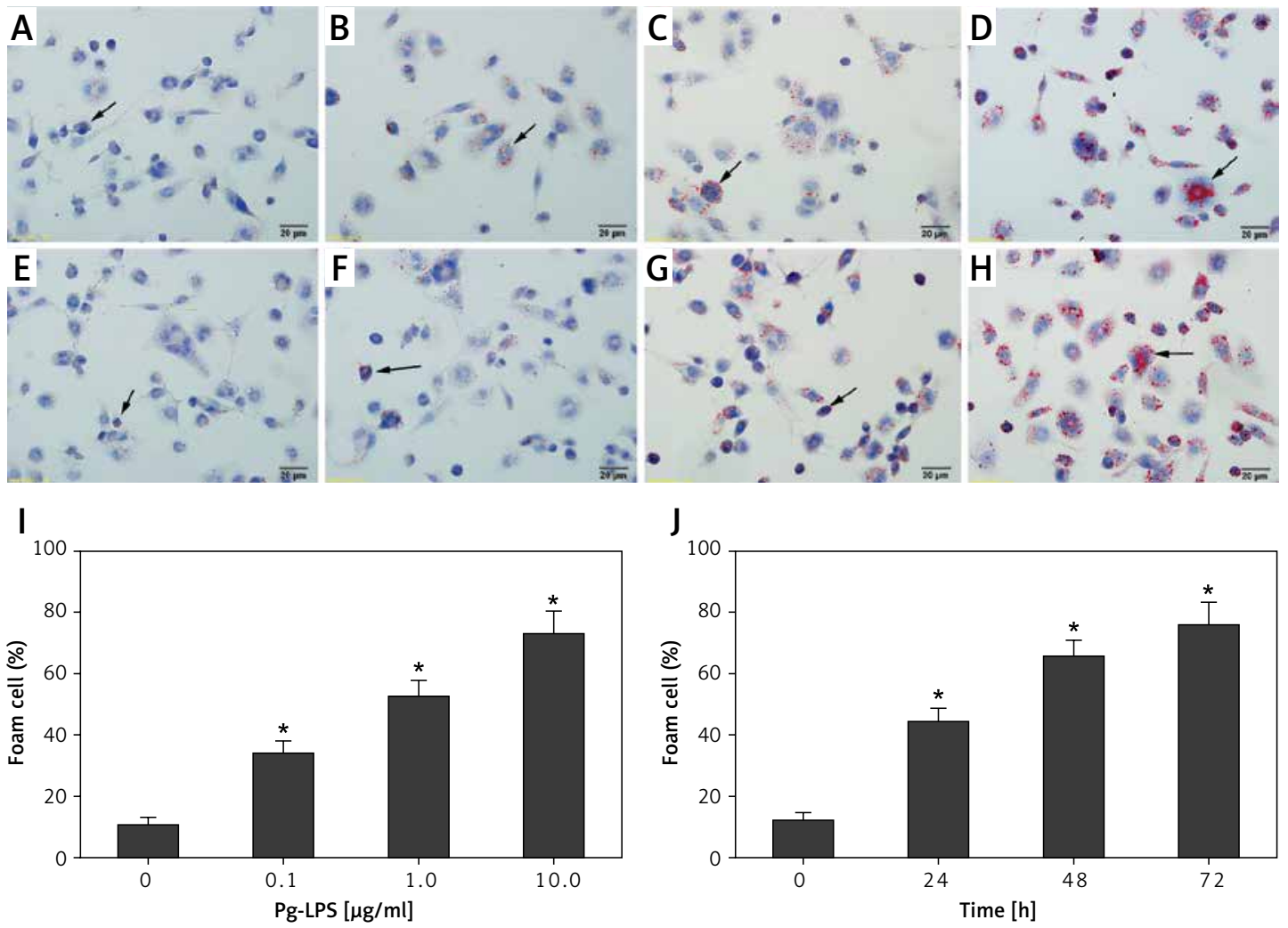

Figure 1. Pg-LPS induced foam cell formation, according to morphological criteria. THP-1-derived macrophages were cultured with or without increasing concentrations of Pg-LPS in the presence of $50 \mu \mathrm{g} / \mathrm{ml} \mathrm{LDL}$ for $48 \mathrm{~h}$. A - Untreated macrophages as a control; B - $0.1 \mu \mathrm{g} / \mathrm{ml}$; C - $1.0 \mu \mathrm{g} / \mathrm{ml}$; D - $10.0 \mu \mathrm{g} / \mathrm{ml}$. I - The number of foam cells induced by different concentrations of LPS. Moreover, incubation of macrophages with LDL $(50 \mu \mathrm{g} / \mathrm{ml})$ and Pg-LPS $(1.0 \mathrm{\mu g} / \mathrm{ml})$ was performed for different durations. E - 0 h as a control; F - 24 h; G - 48 h; H - 72 h. J - The number of foam cells induced by LPS for different durations. Black arrows indicate representative Oil Red $\mathrm{O}$ stained lipid droplets. Original magnification of microphotographs: 400x; scale bar $=20 \mu \mathrm{m}$ 
CD36 mRNA expression dropped to $40 \%$ of that of the control (Figure $4 \mathrm{~A}$ ), while the protein expression fell to less than $40 \%$ of that of the control (Figure 4 B). However, there was no significant time-dependent difference in LOX-1 expression, which was consistent with the results in cells treated with different concentrations of Pg-LPS.

ACAT1 expression was upregulated, whereas ABCG1 expression was downregulated in response to Pg-LPS treatment

In order to determine the effects of Pg-LPS on the esterification of FC into CE and the efflux of FC [7] from macrophages co-cultured with LDL, the expression levels of ACAT1 and ABCG1 were analyzed. As shown in Figure $5 \mathrm{~A}, 10 \mu \mathrm{g} / \mathrm{ml}$ Pg-LPS upregulated ACAT1 mRNA expression to 2.3-fold that of the control, and this effect was concentration dependent. However, ABCG1 expression decreased to only $43 \%$ of that of the control when used at the highest concentration $(10 \mu \mathrm{g} / \mathrm{ml})$. Similar patterns of protein expression were observed by western blotting. At $0.1 \mu \mathrm{g} / \mathrm{ml}$ Pg-LPS, no changes in either ABCG1 or ACAT1 levels were
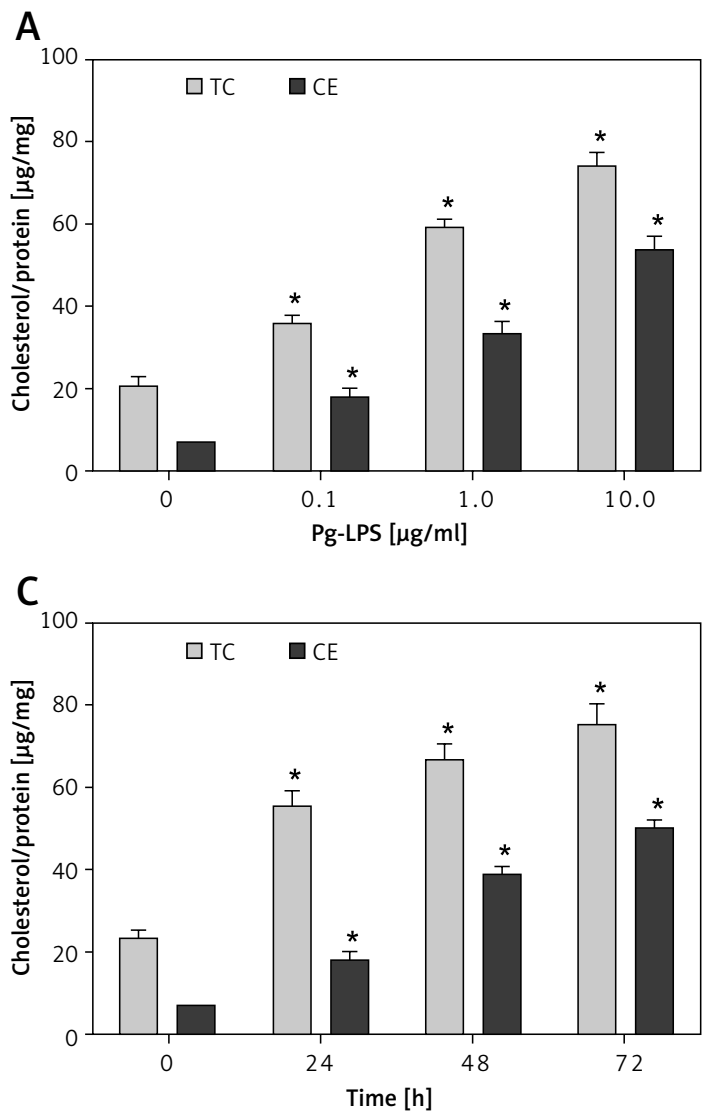

observed $(p>0.05)$. However, the highest concentration of Pg-LPS resulted in approximately 2-fold upregulation in ACAT1 expression and 50\% downregulation of $A B C G 1$ expression (Figure $5 B$ ).

The incubation of THP-1-derived macrophages with $1 \mu \mathrm{g} / \mathrm{ml}$ Pg-LPS and LDL resulted in a maximal 4-fold change in ACAT1 mRNA expression and a 3-fold change in ACAT1 protein expression compared with the control level (Figures $6 \mathrm{~A}$ and $\mathrm{B}$ ). In contrast, $A B C G 1$ expression markedly decreased in a time-dependent manner. Specifically, ABCG1 protein expression was almost completely inhibited, with $93 \%$ reduction at $72 \mathrm{~h}$.

\section{Discussion}

Cumulative evidence published over the last two decades suggests a causal relationship between periodontal infection and cardiovascular diseases (CVDs). Chronic periodontitis is characterized by multiple but transient occurrences of bacteremia, which may result in the systemic spread of LPS from the oral cavity to atherosclerotic plaques, leading to the formation of foam cells. Because of the similarities between

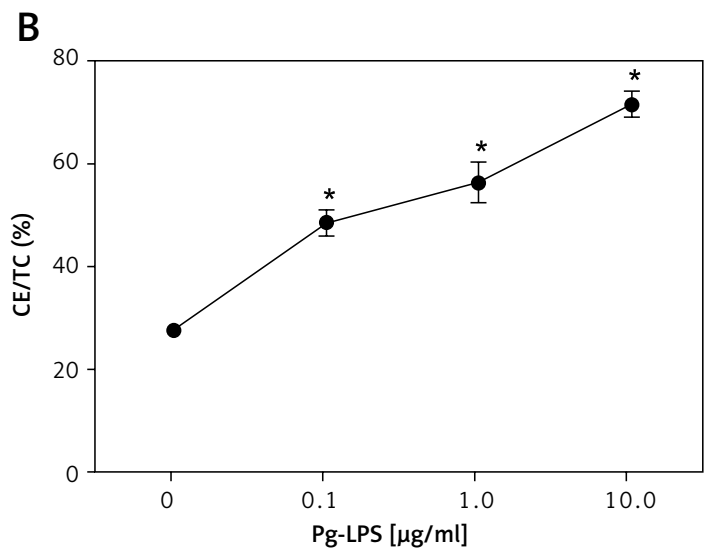

D

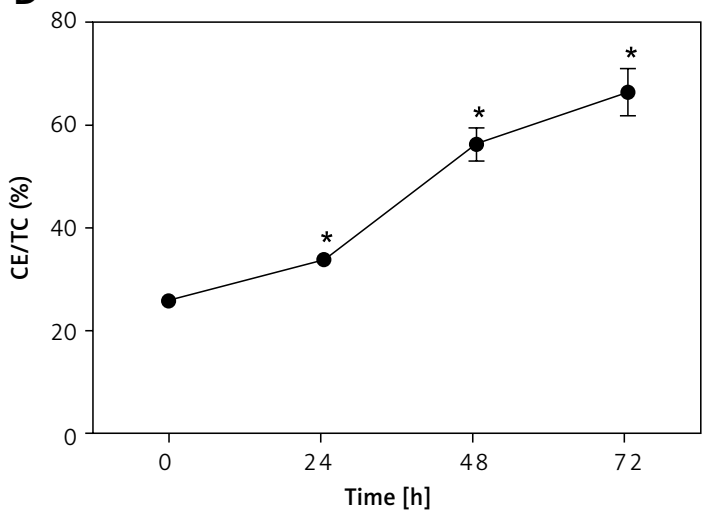

Figure 2. Pg-LPS induced foam cell formation, according to biochemical criteria. Macrophages were exposed to increasing concentrations $(0,0.1,1,10 \mu \mathrm{g} / \mathrm{ml})$ of Pg-LPS for $48 \mathrm{~h}$ in the presence of $50 \mu \mathrm{g} / \mathrm{ml} \mathrm{LDL}(\mathbf{A}$ and $\mathbf{B})$. To measure time-dependent effects, cells were incubated with Pg-LPS $(1.0 \mu \mathrm{g} / \mathrm{ml})$ in the presence of LDL $(50 \mu \mathrm{g} / \mathrm{ml})$ for $0,24,48$, or $72 \mathrm{~h}$ (C and D). A and C - The content of intracellular total cholesterol (TC) and cholesterol ester (CE) was normalized to protein levels and expressed as $\mu \mathrm{g} / \mathrm{mg}$. B and $\mathbf{D}-$ The ratios of CE to TC. Data are presented as means \pm SDs; ${ }^{*} P<0.05$ 
THP-1-derived macrophages and primary peripheral blood mononuclear cell-derived macrophages, THP-1-derived macrophages were exposed to LPS stimuli in the presence of low concentrations of LDL in our study. Our results indicated that PgLPS induced macrophage foam cell formation in a concentration- and time-dependent manner, according to both morphological and biochemical criteria, suggesting that Pg-LPS treatment in-

\section{A}
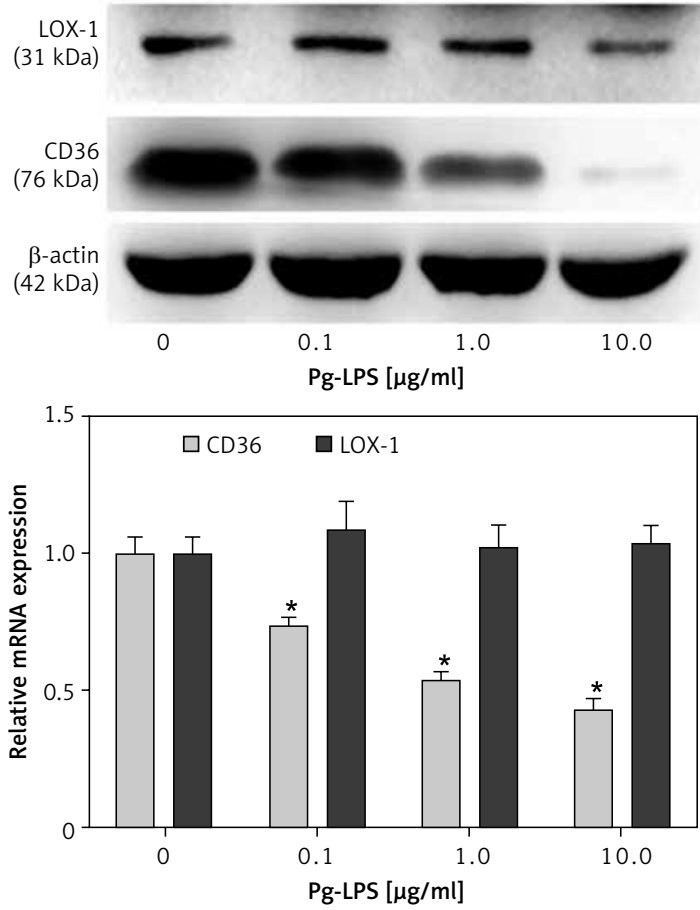

A
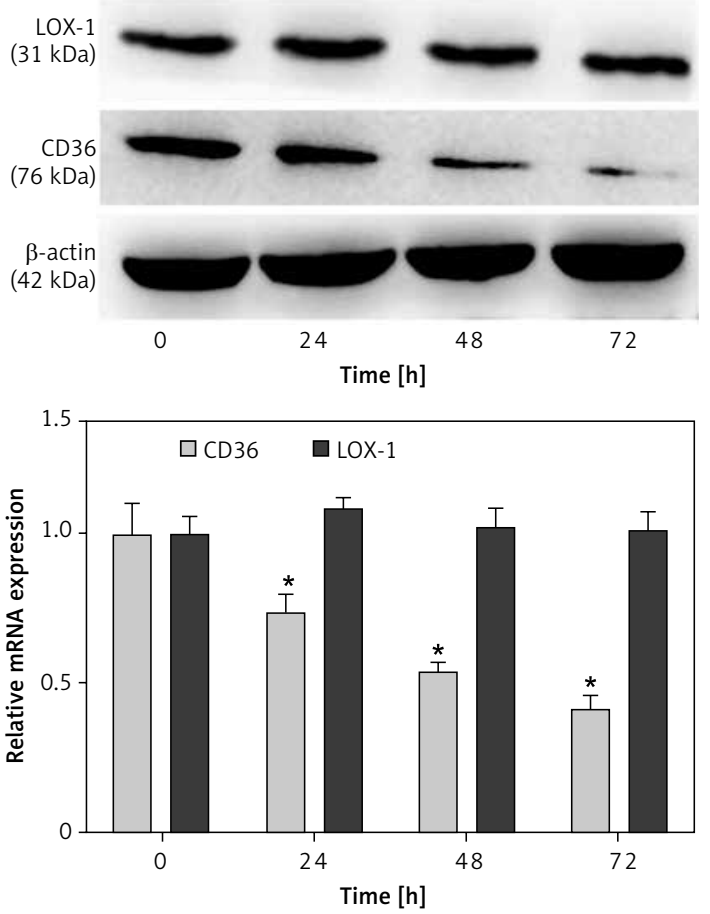

creased the risk of cardiovascular damage. This was consistent with a previous finding that LPS purified from another Gram-negative bacterium, Chlamydia pneumoniae, could induce cholesterol ester accumulation and foam cell formation [19]. The LPS has been shown to increase LDL-R expression, which leads to excessive cholesterol ester accumulation [20]. Additionally, Pg-LPS can enhance the binding of LDL with macrophages and modifi-

B

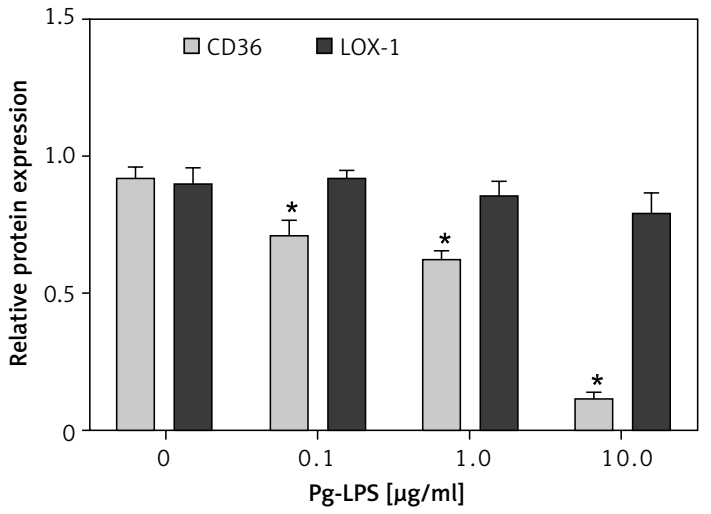

Figure 3. Measurement of concentration-dependent CD36 and LOX-1 expression in Pg-LPS-treated macrophages. Macrophages were exposed to increasing concentrations $(0,0.1,1,10 \mu \mathrm{g} / \mathrm{ml})$ of Pg-LPS for $48 \mathrm{~h}$ in the presence of $50 \mu \mathrm{g} / \mathrm{ml}$ LDL $A-q R T-P C R$ data for CD 36 and $L O X-1$ mRNA expression are presented as fold changes relative to the untreated control $(0 \mu \mathrm{g} / \mathrm{ml})$. B - Western blot analysis of CD36 and LOX-1 protein expression. The protein levels were normalized to those of $\beta$-actin. ${ }^{*} P<0.05$

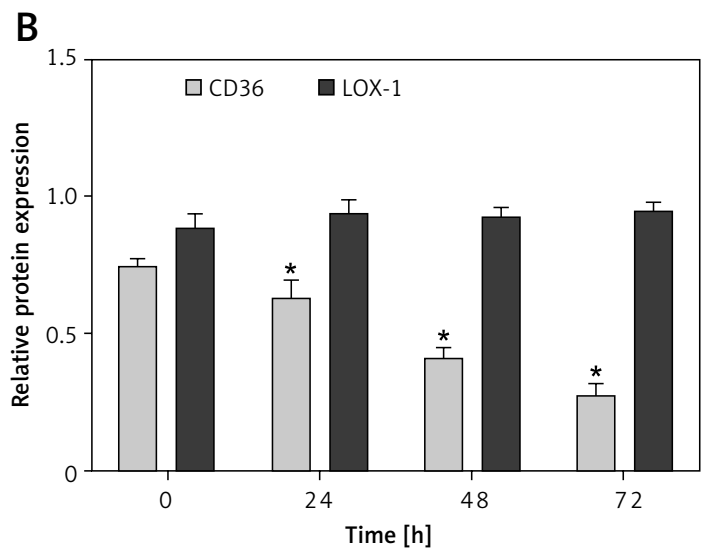

Figure 4. Measurement of time-dependent CD36 and LOX-1 expression in Pg-LPS-treated macrophages. Cells were treated with Pg-LPS $(1.0 \mu \mathrm{g} /$ $\mathrm{ml})$ and $\operatorname{LDL}(50 \mu \mathrm{g} / \mathrm{ml})$ for increasing durations $(0,24,48$, or 72 h). A - qRT-PCR data of CD36 and LOX-1 mRNA expression are presented as fold changes relative to the untreated control $(0 \mathrm{~h})$. B - Western blot analysis of CD36 and LOX-1 protein expression. The protein levels were normalized to $\beta$-actin. ${ }^{*} P<0.05$ 
cation of LDL [7]. Collectively, these data may provide insights into the mechanisms of Pg-LPS-induced foam cell formation in the presence of LDL. Interestingly, in our study, LDL alone did not induce foam cell formation, which was inconsistent with a recent report. The different cell source may cause this inconsistency [21]. Our observation was consistent with another finding that PMA stimulated human monocyte derived-macrophage

A
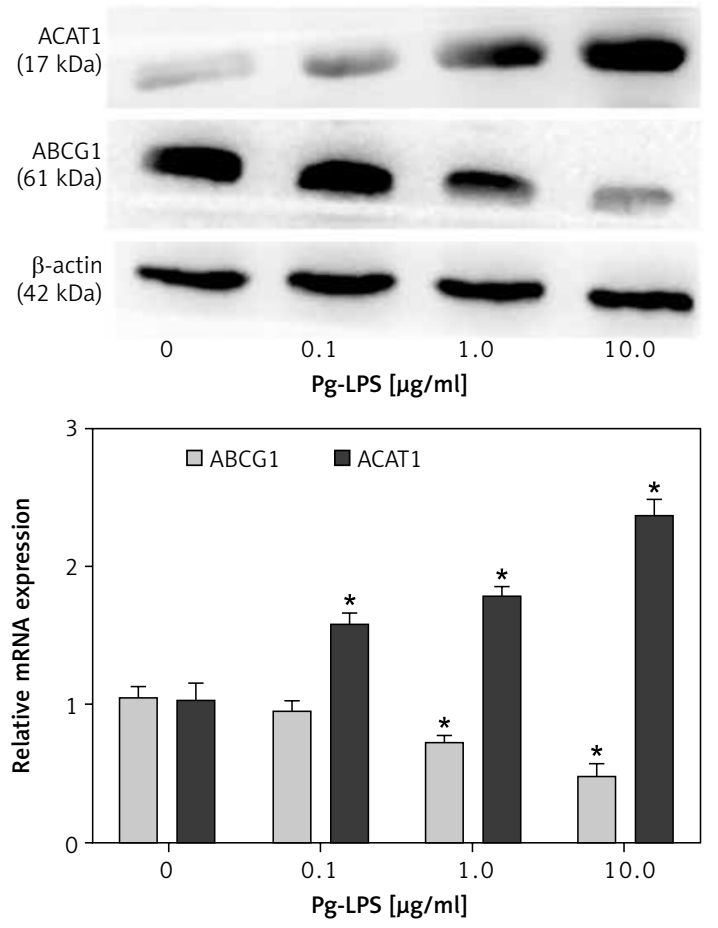

A
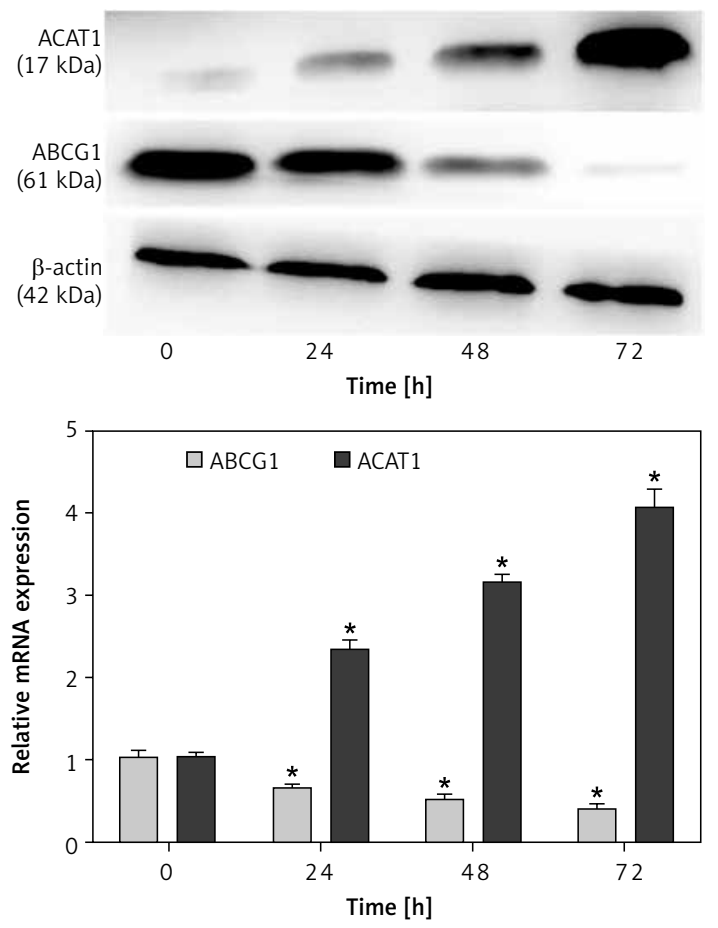

cholesterol accumulation, while there was no significant change in the total cholesterol content without PMA stimulation [22].

Thus, we proceeded to investigate the molecular mechanisms responsible for Pg-LPS-induced foam cell formation. Our study demonstrated that Pg-LPS induced a linear decrease in both CD36 gene and protein expression in a time- and concentration-dependent manner, while no signifi-

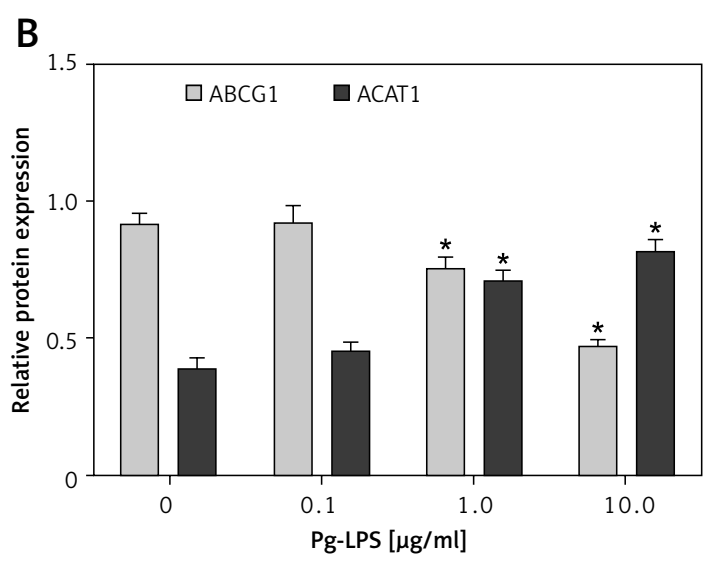

Figure 5. Measurement of concentration-dependent ACAT1 and ABCG1 expression in Pg-LPS-treated macrophages. Macrophages were exposed to increasing concentrations $(0,0.1,1,10 \mu \mathrm{g} / \mathrm{ml})$ of $\mathrm{Pg}$-LPS for $48 \mathrm{~h}$ in the presence of $50 \mu \mathrm{g} / \mathrm{ml} \mathrm{LDL}$. A - ACAT1 and ABCG1 mRNA levels are presented as fold changes relative to the untreated control $(0 \mu \mathrm{g} / \mathrm{ml})$. B - Western blot analysis of ACAT1 and ABCG1 protein expression. The protein levels were normalized against $\beta$-actin as an internal control. Data are presented as means \pm SDs. ${ }^{*} P<0.05$

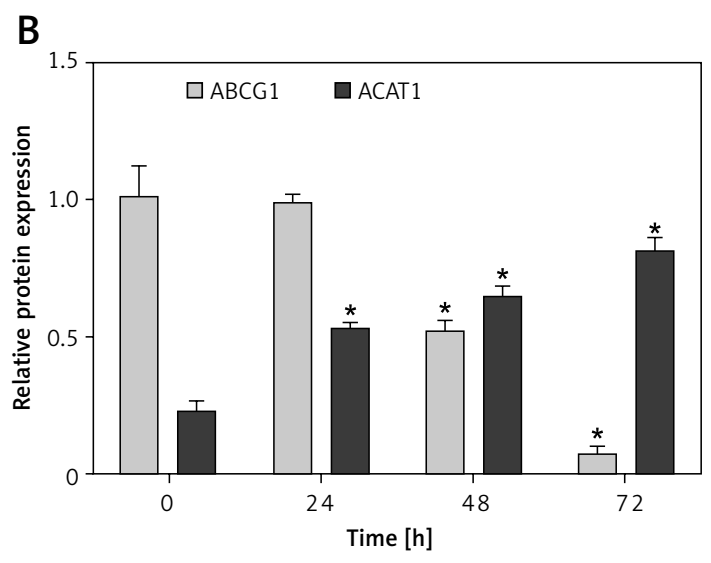

Figure 6. Measurement of time-dependent ACAT1 and ABCG1 expression in Pg-LPS-treated macrophages. Cells were treated with Pg-LPS $(1.0 \mu \mathrm{g} / \mathrm{ml})$ and $\operatorname{LDL}(50 \mu \mathrm{g} / \mathrm{ml})$ for increasing durations $(0,24$, 48, or 72 h). A - ACAT1 and ABCG1 mRNA levels are presented as fold changes relative to the untreated control $(0 \mathrm{~h})$. B - Western blot analysis of ACAT1 and $A B C G 1$ protein expression. The protein levels were normalized to $\beta$-actin as an internal control. ${ }^{*} P<0.05$ 
cant changes were observed in LOX-1 expression. To our knowledge, this is the first study investigating the effects of Pg-LPS on LOX-1. On the other hand, some inconsistent reports describing the effects of LPS on CD36 expression in monocytes and macrophages have been published $[14,23]$. The discordance in CD36 expression found between the previous study [14] and our study could be explained by the presence of LDL in our assays. First, LPS-mediated oxidative stress is known to cause LDL oxidation [24], and ox-LDL reduces LPS-mediated inflammatory effects [25], including CD36 upregulation [26]. Second, LPS has been shown to stimulate LDL-R expression and macropinocytosis in RAW264.7 cells to enhance foam cell formation in the presence of LDL [21]. CD36 downregulation may work as a compensatory mechanism for the excessive uptake of cholesterol-rich LDL via LPS-mediated LDL-R upregulation.

Upon uptake of LDL, excess FC in foam cells can either be re-esterified into CE by ACAT1 or removed from the cells through a receptor-mediated FC efflux pathway regulated by $A B C A 1$ and $A B C G 1$ [8]. Therefore, we further investigated the roles of ACAT1 and ABCG1 in Pg-LPS-induced foam cell formation. To the best of our knowledge, our study is the first to show that in vitro Pg-LPS treatment significantly increased ACAT1 mRNA and protein expression, but inhibited $A B C G 1$ expression in a time- and concentration-dependent manner in THP-1-derived macrophages cocultured with LDL. More notably, LPS-induced foam cell formation and CE accumulation appeared to be associated with the upregulation of ACAT1 and downregulation of ABCG 1, implying that LPS-induced foam cell formation may be influenced by enhanced synthesis of CE via ACAT1 and reduced efflux of cholesterol via ABCG1. No effects of LPS were observed on ABCG1 expression in a previous report [14]. However, our result was consistent with another previous study demonstrating that LPS infection significantly repressed ABCG1 expression [15].

Our previous study demonstrated that Pg-LPS can induce various pro-inflammatory cytokines in macrophages, including tumor necrosis factor (TNF)- $\alpha$, interleukin (IL)-6, and IL-1 [27]. Many inflammatory components participate in atherosclerotic lesion development [28]. However, TNF- $\alpha$ has been demonstrated to specifically enhance ACAT 1 expression to promote atherosclerosis [29]. Therefore, we can speculate that LPS-induced upregulation of ACAT 1 may contribute to the inflammatory conditions produced by LPS.

Additionally, it is possible that ACAT1 upregulation and CD36 downregulation may be a defense mechanism against excess LDL in the culture medium; CD36 downregulation would reduce LDL uptake, and ACAT1 upregulation would help eliminate excess FC generated by LDL.
In conclusion, our study showed that a periodontal infectious agent altered the expression of key molecules regulating lipid uptake and cholesterol esterification and efflux, all of which facilitate the perturbation of cholesterol homeostasis and lead to CE accumulation and foam cell formation. This in vitro evidence further supported the positive correlation between periodontal disease and atherosclerosis via molecular mimicry. Therefore, the prevention and treatment of periodontal infection are of great importance for patients with CVDs.

\section{Acknowledgments}

Fen Liu and Yi Wang contributed equally to this paper.

This work was supported by grants from the National Natural Science Foundation of China (grant no. 81200795) and Zhejiang Provincial Natural Science Foundation of China (grant no. LY12H14003 and no. LQ12H09003).

\section{Conflict of interest}

The authors declare no conflict of interest.

\section{References}

1. Ertek $\mathrm{S}$, Cicero A. Impact of physical activity on inflammation: effects on cardiovascular disease risk and other inflammatory conditions. Arch Med Sci 2012; 8: 794-804.

2. Loesche WJ, Grossman NS. Periodontal disease as a specific, albeit chronic, infection: diagnosis and treatment. Clin Microbiol Rev 2001; 14: 727-52.

3. Humphrey LL, Fu RW, Buckley DI, Freeman M, Helfand $M$. Periodontal disease and coronary heart disease incidence: a systematic review and meta-analysis. J Gen Intern Med 2008; 23: 2079-86.

4. Ford PJ, Gemmell E, Hamlet SM, et al. Cross-reactivity of GroEL antibodies with human heat shock protein 60 and quantification of pathogens in atherosclerosis. Oral Microbiol Immunol 2005; 20: 296-302.

5. Liu B, Cheng L, Liu D, et al. Role of p38 mitogen-activated protein kinase pathway in Porphyromonas gingivalis lipopolysaccharide-induced VCAM-1 expression in human aortic endothelial cells. J Periodontol 2012; 83: 955-62.

6. Yuan Y, Li P, Ye J. Lipid homeostasis and the formation of macrophage-derived foam cells in atherosclerosis. Protein Cell 2012; 3: 173-81.

7. Qi M, Miyakawa H, Kuramitsu HK. Porphyromonas gingivalis induces murine macrophage foam cell formation. Microb Pathog 2003; 35: 259-67.

8. Sekiya M, Osuga J, Igarashi M, Okazaki H, Ishibashi S. The role of neutral cholesterol ester hydrolysis in macrophage foam cells. J Atheroscler Thromb 2011; 18: 359-64.

9. Kunjathoor VV, Febbraio M, Podrez EA, et al. Scavenger receptors class $A-1 / I I$ and $C D 36$ are the principal receptors responsible for the uptake of modified low density lipoprotein leading to lipid loading in macrophages. J Biol Chem 2002; 277: 49982-8.

10. Kataoka H, Kume N, Miyamoto S, et al. Expression of lectin like oxidized low-density lipoprotein receptor-1 in human atherosclerotic lesions. Circulation 1999; 99: 3110-7. 
11. Chang TY, Chang CC, Cheng D. Acyl-coenzyme A:Cholesterol acyltransferase. Annu Rev Biochem 1997; 66: 613-38.

12. Wang H, Germain SJ, Benfield PP, Gillies PJ. Gene expression of acyl-coenzyme-A:cholesterol-acyltransferase is upregulated in human monocytes during differentiation and foam cell formation. Arterioscler Thromb Vascular Biol 1996; 16: 809-14.

13. Kennedy MA, Barrera GC, Nakamura K, et al. ABCG1 has a critical role in mediating cholesterol efflux to $\mathrm{HDL}$ and preventing cellular lipid accumulation. Cell Metab 2005; 1: 121-31.

14. Li XY, Wang C, Xiang XR, Chen FC, Yang CM, Wu J. Porphyromonas gingivalis lipopolysaccharide increases lipid accumulation by affecting CD36 and ATP-binding cassette transporter A1 in macrophages. Oncol Rep 2013; 30: 1329-36.

15. Park Y, Pham TX, Lee J. Lipopolysaccharide represses the expression of ATP-binding cassette transporter G1 and scavenger receptor class B, type I in murine macrophages. Inflamm Res 2012; 61: 465-72.

16. Schaffner T, Taylor K, Bartucci EJ, et al. Arterial foam cells with distinctive immunomorphologic and histochemical features of macrophages. Am J Pathol 1980; 100: 57-80.

17. Gamble W, Vaughan M, Kruth HS, Avigan J. Procedure for determination of free and total cholesterol in microor nanogram amounts suitable for studies with cultured cells. J Lipid Res 1978; 19: 1068-70.

18. Small DM. Cellular mechanisms for lipid deposition in atherosclerosis (first of two parts). New Engl J Med 1977; 297: 873-7.

19. Kalayoglu MV, Byrne Gl. A Chlamydia pneumoniae component that induces macrophage foam cell formation is chlamydial lipopolysaccharide. Infect Immun 1998; 66: 5067-72.

20. Ye $\mathrm{Q}$, Chen $\mathrm{Y}$, Lei $\mathrm{H}$, et al. Inflammatory stress increases unmodified LDL uptake via LDL receptor: an alternative pathway for macrophage foam-cell formation. Inflamm Res 2009; 58: 809-18.

21. Morishita M, Ariyoshi W, Okinaga T, Usui M, Nakashima K, Nishihara T. A. actinomycetemcomitans LPS enhances foam cell formation induced by LDL. J Dent Res 2013; 92: 241-6.

22. Kruth HS, Huang W, Ishii I, Zhang WY. Macrophage foam cell formation with native low density lipoprotein. J Bio Chem 2002; 277: 34573-80

23. Zamora C, Canto E, Nieto JC, Angels Ortiz M, Juarez C, Vidal S. Functional consequences of CD36 downregulation by TLR signals. Cytokine 2012; 60: 257-65.

24. Lopes-Virella MF. Interactions between bacterial lipopolysaccharides and serum lipoproteins and their possible role in coronary heart disease. Eur Heart J 1993; 14: 118-24.

25. Kannan Y, Sundaram K, Aluganti Narasimhulu C, Parthasarathy S, Wewers MD. Oxidatively modified low density lipoprotein (LDL) inhibits TLR2 and TLR4 cytokine responses in human monocytes but not in macrophages. J Biol Chem 2012; 287: 23479-88.

26. Ortiz-Masia D, Diez I, Calatayud S, et al. Induction of CD36 and thrombospondin-1 in macrophages by hypoxia-inducible factor 1 and its relevance in the inflammatory process. PloS One 2012; 7: e48535.

27. Wang Y, Wang H, Ye Q, et al. Co-regulation of LPS and tensile strain downregulating osteogenicity via c-fos expression. Life Sci 2013; 93: 38-43.

28. Kleemann R, Zadelaar S, Kooistra T. Cytokines and atherosclerosis: a comprehensive review of studies in mice. Cardiovasc Res 2008; 79: 360-76.
29. Lei L, Xiong Y, Chen J, et al. TNF-alpha stimulates the ACAT1 expression in differentiating monocytes to promote the CE-laden cell formation. J Lipid Res 2009; 50: 1057-67. 